\title{
Violences à l'acte, violences dans l'acte : pour une histoire des théâtres féministes états-uniens
}

Violence enacted, violence in the act: feminist theatres in the United States since 1970

\section{Madeleine Planeix-Crocker}

\section{(2) OpenEdition}

\section{Journals}

Édition électronique

URL : http://journals.openedition.org/cel/9971

DOI : $10.4000 /$ cel.9971

ISSN : 2262-208X

Éditeur

École du Louvre

Référence électronique

Madeleine Planeix-Crocker, « Violences à l'acte, violences dans l'acte : pour une histoire des théâtres féministes états-uniens ", Les Cahiers de l'École du Louvre [En ligne], 15 | 2020, mis en ligne le 03 novembre 2020, consulté le 05 novembre 2020. URL : http://journals.openedition.org/cel/9971 ; DOI : https://doi.org/10.4000/cel.9971

Ce document a été généré automatiquement le 5 novembre 2020.

\section{(i) $\$$}

Les Cahiers de l'École du Louvre sont mis à disposition selon les termes de la licence Creative Commons Attribution - Pas d'Utilisation Commerciale - Pas de Modification 4.0 International. 


\section{Violences à l'acte, violences dans l'acte : pour une histoire des théâtres féministes états-uniens}

Violence enacted, violence in the act: feminist theatres in the United States since 1970

Madeleine Planeix-Crocker

\section{Des groupes aux troupes féministes : le théâtre comme outil politique}

L'étude des représentations des violence dans l'histoire de l'art invite à une analyse de son incarnation dans des instances performatives ${ }^{1}$. Un retour spécifique sur l'histoire des théâtres féministes états-uniens à partir des années 1970 donne à voir l'émergence d'un genre dramaturgique en lien étroit avec un mouvement politique qui lui est contemporain, à savoir celui du Women's Liberation Movement (WLM). Ce rassemblement féministe, actif à travers le continent nord-américain, émerge en excroissance des mouvements des droits civiques et antimilitaristes en activité dès les années 1960. Pour les mouvements féministes états-uniens, cette période marque une prise de conscience et de parole des femmes au sujet des inégalités de genre, et notamment de la subjugation du corps des femmes et de leur identité sexuelle. Ainsi, le WLM lutte activement contre les violences faites aux femmes et pour leur émancipation, que ce soit dans la sphère publique ou privée. Les revendications de féministes états-uniennes se divisent en deux grandes voies: celle du réformisme juridique pour les féministes dites « libérales » et celle de la libération sexuelle pour les féministes dites "radicales ${ }^{2}$ ». Une méthode de rassemblement exclusivement entre femmes prônée par le WLM se nomme consciousness-raising (CR), éveil de conscience. Pendant ces cercles de parole collectifs, les participantes font le constat non pas du caractère exceptionnel des violences de genre qui marquent leurs expériences de femmes, mais plutôt de leur fréquence qu'elles rapprochent d'une tendance 
« universelle» encouragée par le "patriarcat $»^{3}$. C'est donc dans ce contexte que le slogan « le personnel est politique ${ }^{4}$ » voit le jour et prend son sens.

Parmi les actions politiques du WLM, l'on compte de nombreuses interventions à caractère performatif appelées les zap actions. L'emploi onomatopéique zap souligne l'instantanéité et l'improvisation brandies comme modalités de rassemblement féministe ad hoc dans l'espace public. Un exemple saillant d'une telle action est celui de la manifestation contre le « Miss America Pageant» de 1968, tenue à Atlantic City (NJ, É.-U.). Le communiqué de presse de l'organisatrice, Robin Morgan, une féministe radicale, est marqué par un champ lexical propre au spectacle vivant: " accessoires", « jeu », « scène », « rôles ", " perruques », «farce $~^{5}$. La polysémie des termes cités est percutante, car elle relève à la fois des mises en scène "sociales » que les féministes radicales dénoncent (jeux de rôles assignés et oppressifs, accessoires pour incarner la femme « idéale », etc), mais aussi du théâtre en tant que pratique artistique (composée de pièces, personnages, registres, scénographies). D’ailleurs, parmi les moyens de dénonciation mobilisés par Morgan pour cette manifestation, elle cite : «Il y aura des piquets de grève, du théâtre guerrilla, du tractage, des groupes de lobby pour inciter nos sœurs, candidates à cette farce de concours de beauté, à nous rejoindre ${ }^{6}$ ». Le théâtre disruptif et spontané, qu'elle nomme «théâtre guerrilla », est explicitement référencé comme outil d'intervention par lequel les rôles sociaux vont être (dé)joués par les activistes-comédiennes (fig. 1).

Fig. 1.

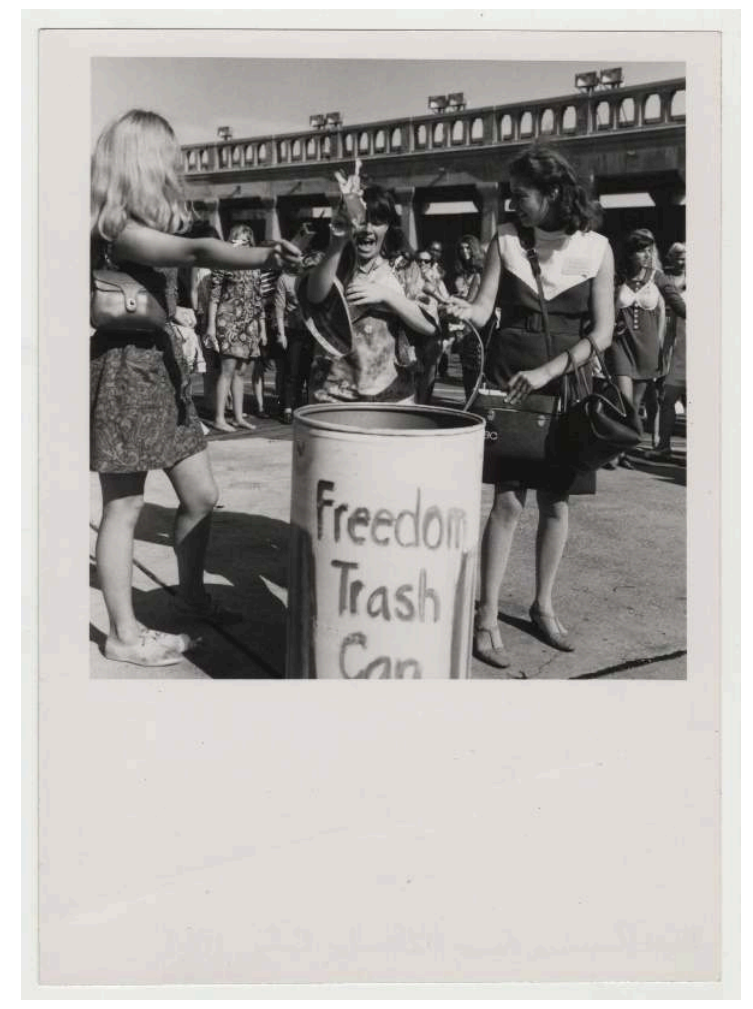

«Freedom Trash Can », manifestation féministe contre le concours de beauté « Miss America », 1968, photographie, Atlantic City, New-Jersey.

(c) Alix Kates Shulman papers, d'après « Women's Liberation Movement Print Culture » Duke University 
Certaines féministes radicales vont au-delà même de ces interventions ponctuelles de soutien à des manifestations politiques, pour en faire une activité professionnelle en soi. En effet, à partir du début des années 1970 se constituent des troupes de théâtre également revendiquées «féministes». Le rapprochement spécifique des troupes au féminisme radical s'illustre par exemple dans le témoignage suivant de Martha Boesing, fondatrice de la compagnie At the Foot of the Mountain :

«il est vrai que nous acceptions, comme le faisait Mary Daly, les catégories qui nous étaient associées : émotionnelles, intuitives, tout comme hystériques [...] Au vu du monde rationnel, linéaire et logique dans lequel nous nous faisions opprimer, ces catégories nous semblaient de bons points de départ pour nos pièces ${ }^{7}$ ».

Biesing évoque l'influence de la théoricienne radicale Mary Daly ${ }^{8}$; elle rejette aussi la linéarité de discours oppressifs faisant écho au projet d'« écriture féminine » de la philosophe française Hélène Cixous ${ }^{9}$. Ainsi, la dramaturge situe plus précisément ces premières troupes de théâtre états-unien dans une continuité du féminisme différentialiste ${ }^{10}$.

5 La ville de New York est le berceau foisonnant de ces rassemblements, et l'on y compte les premières troupes de théâtre féministe états-unien, dont It's All Right To Be Woman (1970-1976) fondée par Sue Perlgut, étudiante en art dramatiques; le Westbeth Playwrights Feminist Collective and Theatre Collective (1971-1975), avec un comité consultatif composé de féministes radicales telles que Gloria Steinem et Florynce Kennedy ; Spiderwoman Theater Troupe (1976-aujourd'hui), fondée par Lisa Mayo et les deux sœurs Gloria et Muriel Miguel, des tribus amérindiennes Kuna et Rappahannock ; et le Women's Experimental Theatre, W.E.T. (1977-1986), fondé par la dramaturge Roberta Sklar. À l'extérieur de la capitale culturelle se montent également des troupes féministes, telles que : la Rhode Island Feminist Theater, RIFT (1973-1985), fondée par Kate Phelps étudiante à Brown University (RI, É.-U.) ; Lilith: Women's Theatre (1974-1985), créée par l'auteure Terry Baum à Berkeley (CA, É.-U.) ; et At the Foot of the Mountain (1974-1991), fondée dans la ville de Minneapolis par Boesing (MN, É.-U.).

\section{La mise en scène de violences : dispositifs et détournements}

6 Les troupes féministes partagent certains points opérationnels avec le WLM tel que le recours aux sessions $\mathrm{CR}$ pendant le processus de création pour cibler des expériences communes et spécifiques aux femmes ${ }^{11}$. Les expériences relevées lors de ces prises de parole partagées sont donc à la fois objet d'étude politique et de création artistique aux objectifs également politiques, donnant lieu à ce que Sue Ellen Case, professeure d'études théâtrales à UCLA (CA, É.-U.), appelle "le théâtre personnel ${ }^{12}$ ». Ce genre artistique inscrit le projet des troupes dans la continuité du programme radical, «le personnel est politique». Les thèmes retenus lors des sessions $\mathrm{CR}$, souvent liés à l'oppression patriarcale manifestée par des violences dans des sphères à la fois publiques et privées, se trouvent ainsi mis en scène. L'on peut penser à Rape-In (1971) $\mathrm{du}$ Westbeth Theatre Collective qui représente une histoire de la vengeance d'une femme violée sur son agresseur; Internal Injury (1978) du RIFT qui traite de cas de violences domestiques ; ou bien encore Cabaret : An Evening of Disgusting Songs and Pukey Images (1979) du Spiderwoman Theater Troupe, une pièce qui critique les représentations violentes de femmes-identifiées diffusées dans la société par le biais 
d'images stéréotypées, commerciales et homogénéisantes. Ainsi, ces troupes s'engagent à illustrer sur scène le continuum de violences de genre ${ }^{13}$ - d'ordre physique et/ou moral, sexuel ou non. Par ces pièces de théâtre, les troupes participent à la visibilité et à la diffusion de sujets qu'elles estiment spécifiquement «féminins " et qui sont jusqu'alors exclus des débats publics. Cet objectif de sensibilisation et de critique sociale s'aligne avec, et par conséquent développe, les actions des féministes radicales du WLM. À l'instar de certaines initiatives du mouvement des féminismes états-uniens, comme celle contre le Miss America Pageant, la présentation des sujets «de femmes » passe par la mise en scène de leurs corps ${ }^{14}$, questionnant leur transformation en objetsfétichistes par le "regard masculin ${ }^{15}$ ». Les pièces qui émergent de thématiques relevées en $\mathrm{CR}$ sont ainsi "taillées d'expériences incorporées ${ }^{16}$ " par les femmes participantes. Le corps devient une ressource à réapproprier par la création artistique. En outre, l'exemple du Cabaret des comédiennes du Spiderwoman Theater Troupe illustre un rejet physique des représentations stéréotypées ainsi que leurs effets sur les corps des femmes, comme l'indique l'emploi du terme puking (vomir) faisant allusion à un acte corporel d'élimination ou de purge (fig. 2 et 3 ).

Fig. 2.

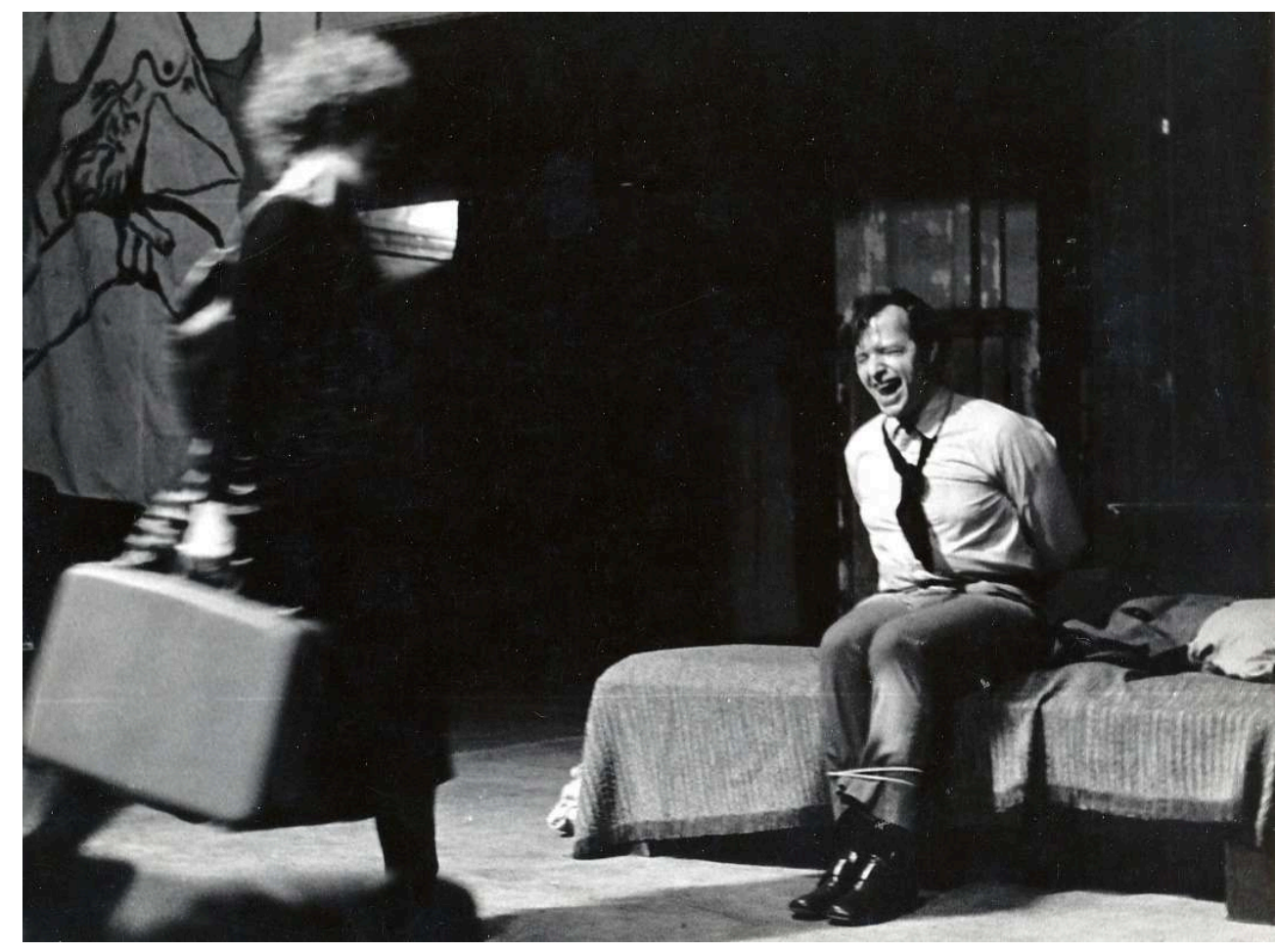

Rape-In, écrit et produit par Westbeth Playwrights Feminist Collective - scène de Liberation de Dolores Walker, I to r, Helen Pugatch et David Kent, 1971, photographie de Paul Lubitz.

(C) Paul Lubitz 
Fig. 3.

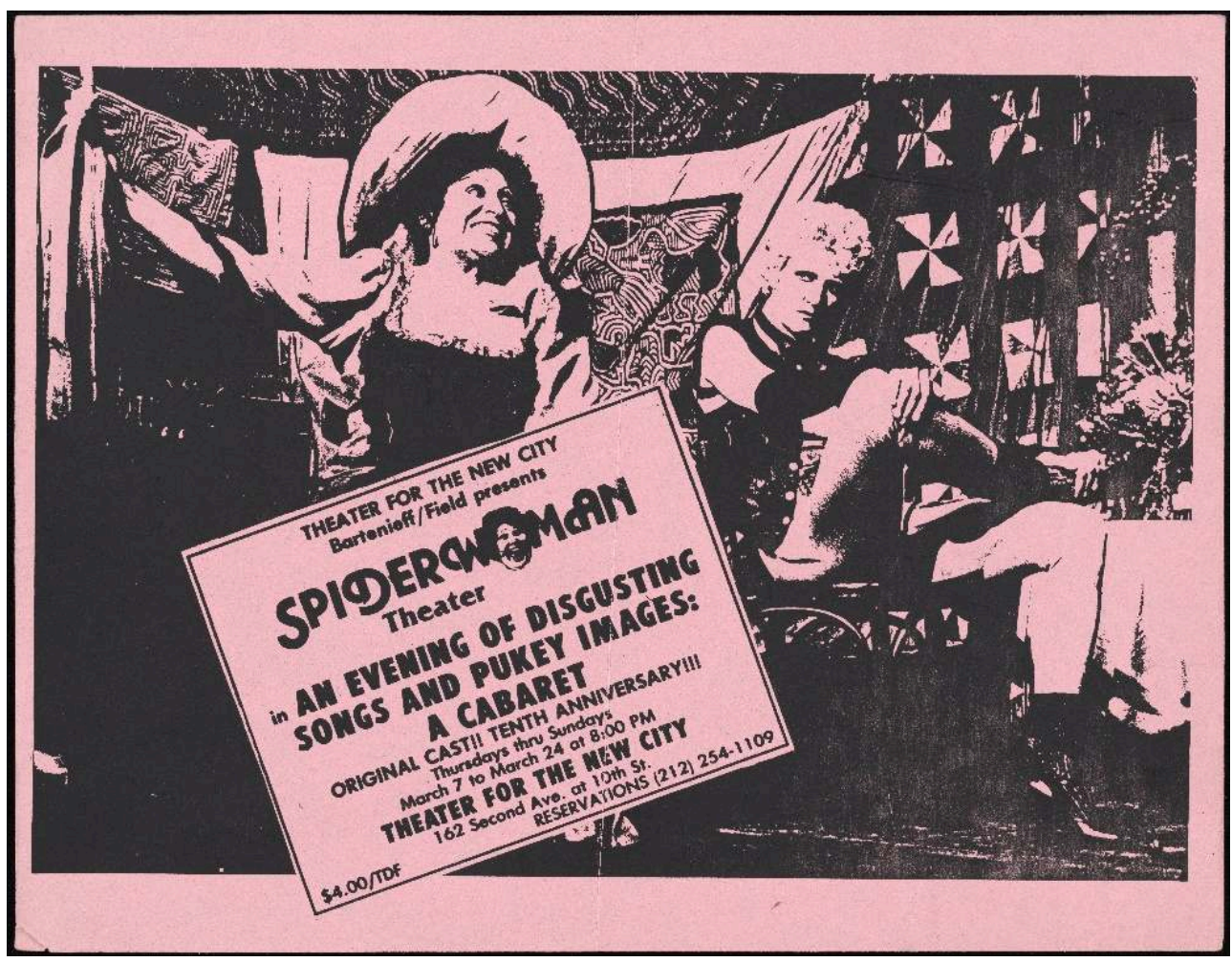

Cabaret, an evening of disgusting songs and pukey images de Spiderwoman Theater, 1979, Affiche de la représentation, New York City, New York.

(c) Spiderwoman Theater

7 De plus, la structuration des troupes féministes en collectifs dé-hiéarchisés fait également écho à celle des groupes radicaux du WLM; la structure collective des compagnies renforce la solidarité entre interprètes ${ }^{17}$. La collaboration devient une ligne directrice du processus de création marqué par l'emploi de l'improvisation, le dialogue et les apports de chaque participante dans les pièces originales ${ }^{18}$. La compagnie Spiderwoman établit sa propre méthode de création collective qu'elle nomme storyweaving (tissage d'histoires) consistant à recueillir les thèmes, idées et rêves de l'ensemble de la troupe qui sont par la suite brodés dans une trame narrative choisie collectivement ${ }^{19}$. L'objectif ultime d'une telle organisation (de la compagnie et de son processus dramaturgique) est la constitution de la troupe comme communauté d'entreaide, à l'instar de la sisterhood (sororité) recherchée par les féministes radicales lors du $\mathrm{CR}^{20}$. Le renforcement des troupes en tant que communauté passe également en partie par le corps, comme en témoigne Harriet Schiffer, membre de la troupe Lilith, à propos de la pratique d'échauffement physique avant des sessions de travail, de répétition ou de représentation: "Ton corps est en harmonie avec ceux des autres participantes. Cela crée un sentiment de communautée ${ }^{21}$. " Les séances d'échauffement et de répétition sont souvent suivies de discussions afin de mettre en mots l'expérience physique vécue en collectivité ${ }^{22}$. Par ailleurs, pour certaines troupes féministes, leur communauté artistique doit refléter celle des rassemblements $\mathrm{CR}$, c'est-à-dire être exclusivement composée de femmes (It's All Right, Spiderwoman, Lilith, At the Foot, RIFT et W.E.T.). Néanmoins, d'autres compagnies œuvrent en mixité (Westbeth Collective). 
8 Les troupes séparatistes souhaitent non seulement se sentir en sécurité pour évoquer des sujets « féminins » lors d'un processus de création, mais aussi effectuer une rupture avec l'« organisation patriarcale du pouvoir ${ }^{23}$ " qu'elles retrouvent dans des compagnies professionnelles non-féministes. En effet, Sklar quitte son engagement en tant que metteuse en scène de la compagnie new-yorkaise Open Theatre, fondée en 1963 par Joseph Chaikin, car elle y repère la même gestion patriarcale que celle dénoncée par les féministes radicales au sein des mouvements de droits civiques. Le spectacle The Mutation Show (1973) marque sa dernière mise en scène pour l'Open Theatre. La pièce traite de la socialisation d'une petite fille née parmi les loups, puis de son adoption par des missionnaires qui la domptent en «femme». Au sujet de cette expérience, Sklar remémore ainsi une autre forme de violence issue de la discrimination dans le milieu professionnel du théâtre :

«Jusqu'à cette pièce, j'avais l'impression d'être l'égale des hommes de la compagnie, même si je savais que la société n'en pensait pas moins pour autant. Lorsqu'il s'agissait de reconnaître des inégalités sociales contre les femmes, mes collègues-hommes ne pouvaient plus les considérer comme des problèmes politiques. [...] Mon travail n'était pas reconnu dans la troupe et je me suis ainsi tournée vers des compagnies féministes ${ }^{24}$.»

9 Après son départ de l'Open Theatre, Sklar fonde la Women's Unit (1972) avec des étudiantes de son cours de théâtre à Bard College (NY, É.-U.) ; les études théâtrales vont ainsi à la rencontre des women's studies (études des femmes et féministes) établies en milieu universitaire états-unien dès 1970.

10 Il faut également souligner que de tels choix de gestion, motivés par des convictions politiques, sont également impulsés par une réalité économique, à savoir l'inégalité d'accès des femmes aux scènes et aux subventions nationales. Helen Krich Chinoy, historienne de théâtre états-unien, dénonce cette disparité qui entraîne une forte précarité parmi les dramaturges et interprètes femmes : « des dramaturges femmes ont été retenues dans l'avancée de leur carrière par un a priori qu'elles sont incapables de prendre des décisions financières et artistiques seules ${ }^{25}$.» Ces inégalités professionnelles fondées sur le genre sont « officiellement » reconnues dès 1963 lors de la publication du rapport American Women publié par le «Presidential Commission on the Status of Women » $(\mathrm{PCSW})^{26}$. Les disparités se révèlent répandues dans l'ensemble du milieu du travail états-unien. Les troupes féministes se voient dans l'obligation de recourir aux méthodes do it yourself (DIY) du fait maison pour la création de costumes, de décors ou de documents de communication pour la promotion de spectacles. L'éloignement des troupes féministes des scènes subventionnées influence également en partie les lieux de représentation de leurs pièces. Effectivement, faute de moyens financiers et de reconnaissance professionnelle, les compagnies ont recours à des espaces alternatifs tels que des églises abandonnées, des parcs ou bien des cafés ${ }^{27}$. Bien que l'acte du performing elsewhere ${ }^{28}$ (représenter ailleurs/représenter l'ailleurs) ne soit pas exclusivement le propre des troupes féministes, comme le note Elaine Aston, professeure d'études théâtrales à Lancaster University (R.-U.), le recours à cette pratique par nécessité est représentatif des réalités économiques très précaires de ces compagnies. Le corps des femmes - comme sujet abordé et comme outil de représentation des comédiennes - est ainsi souvent situé sur une scène tout simplement vide ; leur message résonne d'autant plus fort.

11 Ces choix, amenés par volonté ou par nécessité, marquent donc une rupture des troupes féministes radicales avec le fonctionnement habituel des compagnies 
théâtrales états-uniennes. Néanmoins, en continuité avec la pratique théâtrale traditionnelle, les représentations des troupes féministes s'effectuent pour un public. Ainsi, de quelle manière celles-ci se distinguent-elles d'un accueil «classique » des spectatrices et spectateurs? Tout d'abord, le CR est désormais adopté comme méthode de création des troupes féministes radicales dans le but de constituer une communauté de collaboratrices et collaborateurs; or, une fois les résultats du CR mis en scène, l'objectif d'éveil de la conscience se voit étendu aux membres du public. Pour ce faire, le choix des registres de représentation est également important. En effet, il ne s'agit pas simplement de partager des expériences de violence relevées pendant le $\mathrm{CR}$, mais de les rendre accessibles et identifiables au public. Ce faisant, les troupes espèrent prolonger l'expérience de leur prise de conscience aux spectatrices et spectateurs. Dans un premier temps, ceci passe par l'emploi du réalisme dans la représentation théâtrale des féministes radicales, en opposition à la distanciation prônée par le dramaturge allemand Bertolt Brecht, dans l'objectif d'éviter l'identification du public aux interprètes ${ }^{29}$. Selon les troupes radicales, les expériences de «femmes » partagées dans la communauté d'interprètes doivent être transmises avec vraisemblance au public. Ceci implique également de traduire sur scène les registres émotionnels dégagés lors des sessions de CR. La colère est une expression prégnante des rassemblements, considérée une « émotion productive à partir de laquelle créer du théâtre ${ }^{30}$ ».

12 Par ailleurs, concernant la représentation spécifique de violences faites aux femmes, certaines troupes ont recours à d'autres registres aisément reconnaissables par le public et qui facilitent la transmission d'un message sinon traumatique, du moins douloureux. En effet, selon Noelia Hernando-Real, spécialiste de littérature américaine à l'Universidad Autónoma de Madrid, la comédie et l'humour sont des stratégies déployées par des dramaturges femmes lors de la représentation de situations de violence. Hernando-Real soutient que le rire peut être plus efficace que les pleurs pour éveiller la conscience du public et faciliter une réparation collective ${ }^{31}$. L'on peut se rappeler ici du Cabaret de Spiderwoman Theatre, par exemple, où l'emploi de la farce provoque le rire mais dont l'objectif ultime est de permettre au public d'identifier et de s'identifier aux événements de violences représentées sur scène.

13 En outre, dans un prolongement de la méthode $\mathrm{CR}$ qui souhaite favoriser la libre expression dans un groupe de soutien entre femmes, certaines troupes tentent d'établir un environnement attentif aux besoins du public. Par conséquent, des compagnies mettent en place une politique tarifaire accessible, ainsi qu'une garde d'enfants le temps des représentations ${ }^{32}$. La tentative de création d'un tel espace de protection peut parfois passer par le choix de représenter des spectacles devant un public constitué uniquement de femmes ${ }^{33}$. Ainsi, les troupes féministes radicales états-uniennes élaborent un travail artistique qui, de par sa méthodologie et ses thématiques, reflète les revendications portées par le WLM. L'art se met au service de cet objectif politique, mais devient également un moyen de faire de la politique. Comme vu précédemment, un tel travail implique l'invention de nouvelles pratiques, souvent en avec des champs traditionnels de théorisation ou - concernant plus spécifiquement le théâtre - de création. 


\section{Pour un genre nouveau et des subjectivités à l'acte}

14 À partir de la fin des années 1970, Case reconnaît en effet l'émergence d'un genre artistique nouveau issu des pièces de troupes féministes qui se voient progressivement influencées par les apports critiques du champ de la théorie linguistique féministe ${ }^{34}$. En effet, en tentant de représenter la catégorie « femme » comme « Sujet » signifiant et non pas signifié par les discours symboliques et le regard masculin, les troupes féministes ont recours à une sémiotique "féminine». L'ambition est de comprendre de quelle manière le théâtre peut représenter leur condition de «femme». À l'instar des théoriciennes différentialistes qui interviennent au sujet des discours phallocentriques, certaines troupes féministes radicales reviennent au répertoire du théâtre classique pour cibler la complicité de canons dramaturgiques dans l'aliénation de la femme comme Sujet-protagoniste ${ }^{35}$. C'est le cas de Spiderwoman Theatre, par exemple, qui effectue une adaptation originale, The Lysistrata Numbah (1977), reprenant l'œuvre du $\mathrm{V}^{\mathrm{e}}$ siècle avant J.-C. d'Aristophane pour présenter une lecture féministe des relations de pouvoir qui la constitue; ou bien encore de la compagnie W.E.T. qui produit Electra Speaks (1980) pour redonner une voix - par le biais de nouveaux écrits de femmes - aux personnages féminins de la famille mythologique des Atrides, livrés jusqu'alors à des destins alloués par un auteur masculin, Sophocle, au V siècle avant J.-C. ${ }^{36}$. Ce faisant, les compagnies se rapprochent d'un programme de théorie critique féministe proposé par Teresa de Lauretis, professeure émérite à University of California Santa Cruz (CA, É.-U.), à savoir celui de «re-raconter des histoires connues afin de déstabiliser les mythes littéraires originel ${ }^{37}$ ». En se rapprochant notamment du travail de Cixous dont elle salue le projet d'écriture féminine, Case soutient que le travail initié par les troupes féministes radicales aux États-Unis pourrait être étudié comme une "nouvelle forme féminine du théâtre ${ }^{38}$ ». La chercheuse reconnaît de même la remise en question de cette théorisation linguistique par la pensée féministe poststructuraliste de la fin des années 1980 qui tente de déconstruire les catégorisations "femme » et "féminine " pour en démontrer le potentiel de subversion face aux discours qui les ont produits ${ }^{39}$. Il revient donc à étudier les implications de cette théorie critique poststructuraliste sur les pratiques théâtrales féministes états-uniennes.

La remise en question des objectifs du WLM par la génération politique émergente des années 1980 mène à une déstabilisation de certains groupes de féministes radicales. Cette évolution, en faveur de la déconstruction de catégorisations et de la considération de nouvelles subjectivités aux identités plurielles et fluides, touche aussi les troupes féministes radicales dont l'identité constitutive est à l'image de celle des groupes radicaux, à savoir majoritairement blanche et de classe moyenne, éduquée. Les compagnies en question commencent à se dissoudre vers la fin des années 1980 comme on peut le remarquer en étudiant leurs dates d'activité. La tension qui s'opère au sein des troupes radicales peut être expliquée par des difficultés structurelles présentes dès leurs origines :

«Ces groupes n'avaient pas les mécanismes pour négocier les différences d'entre les femmes car leur projet politique et artistique était fondé sur le principe du consensus et n'était pas en mesure de proposer des perspectives variées, sans que cela implique des changements fondamentaux dans leur fonctionnement ${ }^{40}$. "

Cette évolution n'équivaut pas à la fin du genre artistique mais incite à sa réorientation, marquée par l'apparition de nouvelles méthodologies et formes de représentation désormais portées par des artistes représentatives des nouvelles subjectivités 
revendiquées dans la théorisation des féminismes ${ }^{41}$. Un programme de mise en pratique de cette nouvelle théorisation par l'art est déjà initié par la "théorie performative du genre » de la philosophe états-unienne Judith Butler qui fait référence au caractère « performatif » du genre, tout en invitant à une intervention subversive par la création artistique et notamment par le théâtre ${ }^{42}$. En effet, cette nouvelle génération de créatrices engagées est fortement influencée par les théorisations post-coloniales, queer et intersectionnelles ${ }^{43}$, que l'on retrouve sur scène. Par ailleurs, cette génération apparaît à un moment de backlash ${ }^{44}$ politique contre les féminismes états-uniens, notamment porté par les gouvernements des présidents Ronald Reagan (1981-1989), puis George H. W. Bush (1989-1993). Ce contexte va aussi agir sur l'évolution du genre artistique. L'action double du conservatisme politique et de la propagation du néolibéralisme mène à des revendications de politiques identitaires passant par une manifestation de l'individualité, en opposition à la mobilisation communautaire lancée par la poétesse féministe et radicale Audre Lorde: "Sans communauté, il n'y a pas d'émancipation, mais seulement l'armistice la plus vulnérable et temporaire entre un individu et son oppresseur ${ }^{45}$ ». En réponse à ce courant politique, le genre autobiographique trouve une place particulièrement importante en matière de production culturelle revendiquée féministe, ce qui se traduira notamment au théâtre par l'emploi du monologue et du seule en scène ${ }^{46}$.

\section{Perspectives contemporaines : les cas de Holly Hughes et des Monologues du vagin}

17 À partir des années 1980, le travail de la dramaturge et interprète féministe Holly Hughes incarne ce tournant critique et artistique. Privilégiant la forme du seule en scène, Hughes présente sa vie et les violences sociales qu'elle a subies en tant que femme-identifiée lesbienne. En effet, ses monologues sont composés à partir d'expériences personnelles; Hughes s'inscrit alors dans le genre du théâtre autobiographique féministe selon Jill Dolan, professeure d'études théâtrales à Princeton University (NJ, É.-U. $)^{47}$. Lynda Goldstein, professeure d'études culturelles à Pennsylvania State University (PA, É.-U.), soutient de plus que le travail de Hughes se rapproche du récit confessionnel; or la confession est performative selon Butler, tout comme l'est le genre ${ }^{48}$. Ainsi, re-présenter des éléments autobiographiques par l'emploi continu et répétitif de la confession trouble les structures identitaires hégémoniques ${ }^{49}$. Ce faisant, la dramaturge se re-présente comme le «sujet excentrique " articulé par Lauretis, dont la lesbienne est l'incarnation emblématique ${ }^{50}$. Par ce choix, Hughes se positionne en défiance face aux représentations normées et violentes du corps de femmes-identifiées, et notamment de celles des lesbiennes qui expriment des désirs à l'encontre de l'hétérosexualité compulsive dénoncée par Butler. À ce titre, Hughes se décrit comme une "artiste de l'évasion ${ }^{51}$ ", fuyant une adolescence contrainte vécue dans une région rurale ultra-conservatrice du Michigan, qui donne lieu à son premier monologue parodique, intitulé Well of Horniness (1985). Toutefois, en arrivant à New York, Hughes se retrouve de nouveau à fuir des organisations patriarcales dans le milieu théâtral, comme celles dénoncées auparavant par Sklar. Par conséquent, à l'instar des troupes radicales, ses pièces sont présentées dans des espaces alternatifs de représentation; elle se fait connaître notamment au "Women's One World (WOW) Café » à New York, un café-théâtre féministe fondé par Lois Weaver et Peggy Shaw du 
duo de théâtre lesbien Split Britches ${ }^{52}$, qui produit notamment le travail de femmesidentifiées (straight et lesbiennes, bisexuelles, trans et queer) et de dramaturges de couleur $^{53}$. Parmi d'autres monologues de Hughes, on peut citer: The Lady Dick (1984), World Without End (1989) et Clit Notes (1996). Le travail de la dramaturge contre les violences de genre est annoncé d'emblée à travers les titres de ses pièces, et passe également par une réhabilitation linguistique d'un vocabulaire péjorativement assignée aux femmes-identifiées ${ }^{54}$. Goldstein rejoint Dolan, en défendant que les monologues de Hughes ne ré-articulent pas sur scène la production et le contrôle du plaisir des femmes par des systèmes de pouvoir, mais proposent une re-signification de la dynamique du plaisir et du dé-plaisir entre des corps et subjectivités "différents", car hors des normes hétérosexuelles ${ }^{55}$. Ainsi, les pièces de Hughes ne la re-signifient pas en tant qu'objet du regard masculin. Effectivement, en reprenant l'adage de la théoricienne féministe Monique Wittig «les lesbiennes ne sont pas des femmes ${ }^{56}$ »-qui tente de situer la lesbienne hors-champ de la catégorie "femme » en tant que produit des hommes, du fait de son identité sexuelle dé(f/v)iante-, elle n'est toujours pas « femme » une fois sur scène ${ }^{57}$ (fig. 4).

Fig. 4.

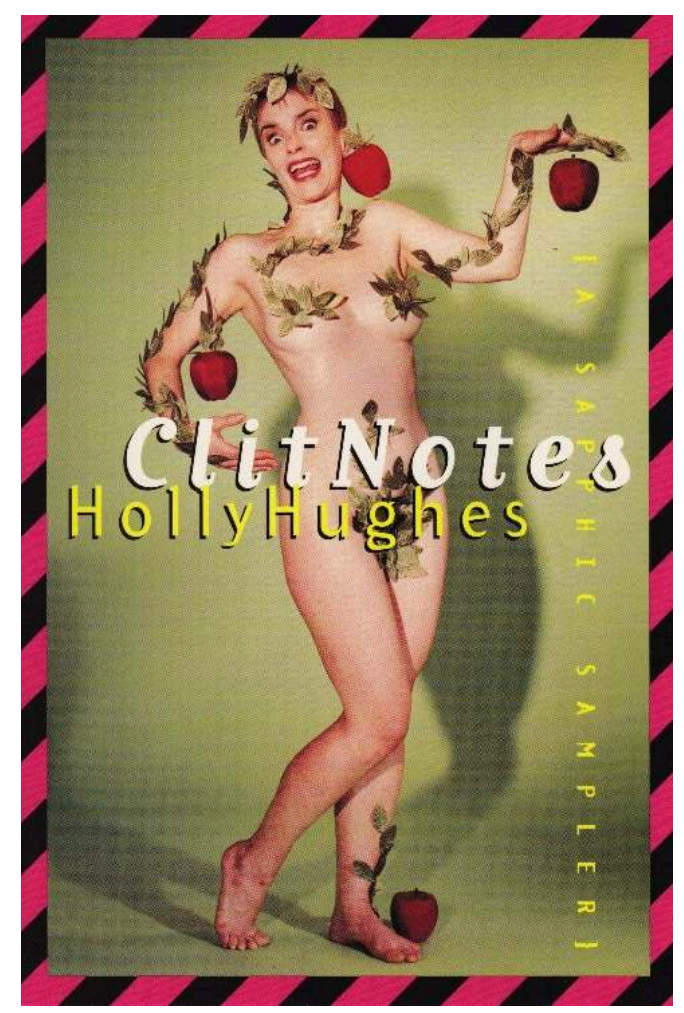

Clit notes - a sapphic sampler, Holly Hughes, 1996, Grove Press, New York City, New York.

(c) John Lovett

Toutefois, la nature pornographique et interdite du travail de Hughes fait l'objet de scandale en 1990 lorsque John Frohnmeyer, directeur du National Endowment for the Arts (NEA) ${ }^{58}$, annule quatre bourses dont une allouée à la dramaturge. Frohnmeyer se défend avoir agi sous l'ordre direct du président George H.W. Bush qui aurait condamné le « contenu blasphématoire ${ }^{59}$ " du travail des artistes en question, adoptant le nom des « NEA 4 ». Ce débat s'inscrit dans le cadre d'une culture war (guerre de la culture) états- 
unienne qui érige une idéologie conservatrice et fortement religieuse contre des valeurs progressistes, telles que le soutien fédéral des arts prélevé à partir d'impôts ${ }^{60}$. Les NEA 4 mènent le combat - qu'elles et ils qualifient de censure à l'encontre du premier amendement constitutionnel ${ }^{61}$ - jusqu'à la Cour Suprême et reçoivent gain de cause (ainsi que la restitution de leurs bourses) en 1993. Sortant des interstices politiques, culturels et sociaux auxquelles elles se voient livrées, ces nouvelles subjectivités se trouvent toujours en ligne de mire de discours normés (notamment, au nom de la loi américaine qui protège la moralité publique). Dans le but de subvertir ce procès largement médiatisé, Hughes écrit le seule en scène Preaching to the Perverted (1999) où elle décentre le débat national autour de questions de liberté, de citoyenneté et de démocratie, avec une perspective queer de sujet excentrique, marquant ainsi une intervention politique par le corps lesbien. Au sujet de cette pièce qui retrace le déroulement du procès, Dolan défend qu'assigner le travail de Hughes au genre comique est trop réducteur et risque de « priver la performance de son geste critique en tant que performance utopique ${ }^{62}$ ». En effet, le solo de Hughes est un appel urgent à l'action qui engage également les spectatrices et les spectateurs. À ces fins, l'interprète échange avec les publics lors de discussions post-performance afin de revenir sur les événements de violence qu'elle décrit sur scène. Ce faisant, la dramaturge rappelle que la performance est un " espace à part ", tout en restant une pratique engagée et liée à la sphère publique dans laquelle l'agentivité mutuelle de l'interprète et des spectatrices-spectateurs peut faire sens ${ }^{63}$. Le sens de la pièce et celui de son action politique qu'elle invoque se co-construisent au moment même de la représentation. Cette intervention est d'autant plus importante que, comme le défend Goldstein, « les confessions performatives et (out)rageuses d'artistes femmes-identifiées, telles que Hughes, seront toujours contrées et appropriées par d'autres discours indignés issus de la culture publique états-unienne ${ }^{64} »$. Pour Hughes, ses pièces deviennent ainsi une stratégie de survie pour lutter contre les violences de genre subies dans sa vie.

La pièce The Vagina Monologues d'Eve Ensler, créée en 1996 et publiée en 1998, marque un deuxième exemple d'intervention féministe et postmoderniste par le théâtre, apportant une attention particulière à la présentation des enjeux de violences de genre, notamment d'ordre sexuel. En effet, les Monologues sont inspirés de deux cents entretiens de femmes-identifiées au sujet de leur anatomie génitale, qui agit comme «métaphore » selon l'auteure, de leur vie ${ }^{65}$. Comme son titre l'indique, l'œuvre revient sur des sujets tabous, abordés par les troupes radicales des années 1970, tout en les adaptant aux évolutions de représentation théâtrale portées par des dramaturges telles que Hughes, notamment par le recours au monologue. La pièce s'inscrit dans la période historique que Camille Froidevaux-Metterie, professeure de science politique à l'université de Reims, identifie comme moment charnière du mouvement féministe «occidental » qu'elle nomme le "tournant génital ${ }^{66}$ ». Les Monologues deviennent un phénomène national puis international: l'œuvre est traduite en 48 langues et fait l'objet de représentations à travers le monde. La diffusion de la pièce est encadrée par V-DAY, l'association à but non lucratif fondée par Ensler en 1999. L'engagement de la dramaturge contre les violences faites aux femmes et filles-identifiées prend donc les théâtres féministes comme point de départ à une action politique qui s'étend bien audelà du plateau, apportant son soutien à des actions sociales. Les textes d'Ensler sont désormais incarnés par des artistes-activistes partout dans le monde, allant d'étudiantes et étudiants en université, à des troupes amatrices et professionnelles. 
Fig. 5.

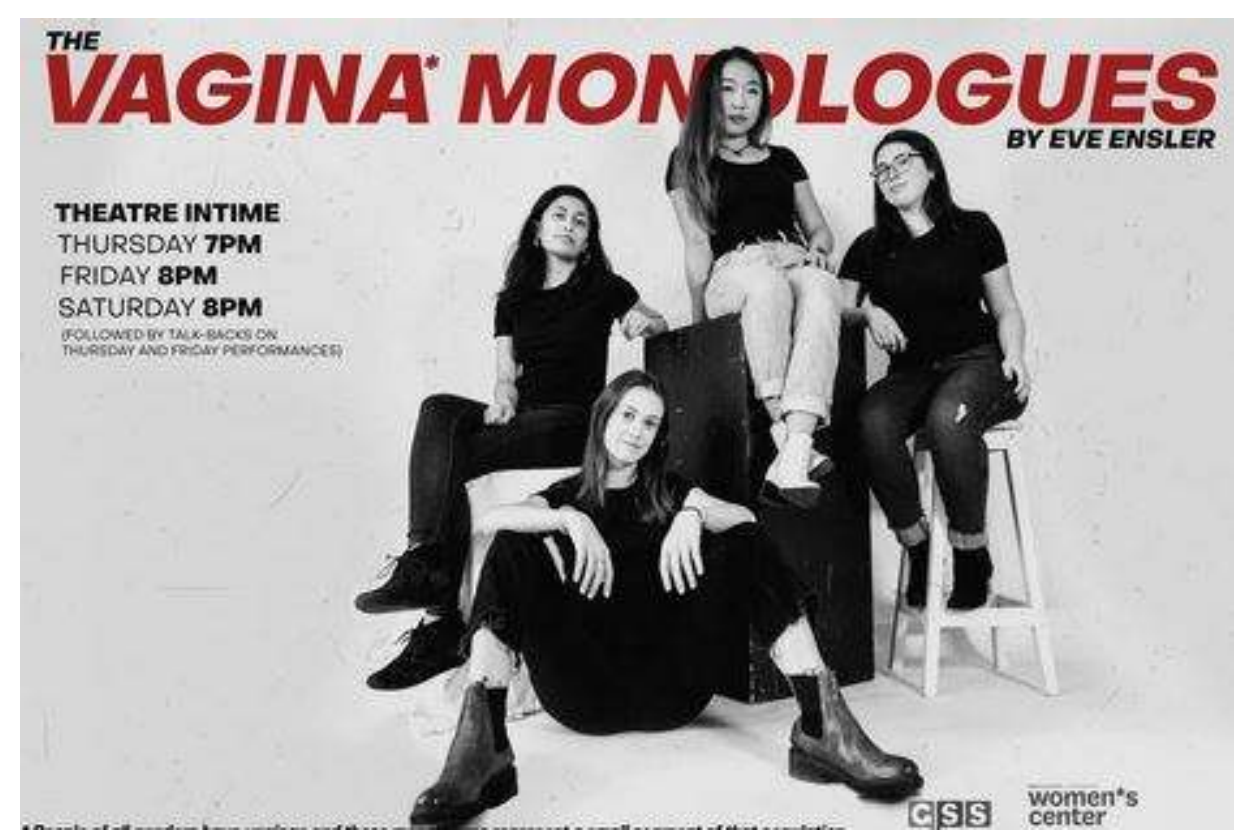

The Vagina Monologues, Eve Ensler, Affiche de la représentation donnée à Princeton University, 2019, Princeton, New Jersey.

(c) The Vagina* Monologues

Ainsi, l'analyse des théâtres féministes états-uniens à partir des années 1970 permet de revenir sur des représentations sur scène de violences de genre dans l'objectif d'inciter un engagement politique des spectatrices et des spectateurs, ainsi que de réhabiliter par le processus d'historicisation ces pratiques artistiques reléguées aux marges trop souvent ignorées des féminismes.

\section{NOTES}

1. L'emploi de l'écriture inclusive a ici été retiré à la demande du comité éditorial. Nous nous plions à cette demande, sans pour autant $\mathrm{y}$ adhérer.

2. Jo Freeman, «The Origins of the Women's Liberation Movement », American Journal of Sociology, vol. 78, n 4, 1973, p. 795.

3. Shulamith Firestone, The Dialectic of Sex: The Case for a Feminist Revolution, New York, Morrow, 1970, pp. 10-11.

4. Carol Hanisch, «The Personal is Political », Notes from the Second Year: Women's Liberation, New York, Ed. Shulamith Firestone [Radical Feminism], 1970. Accessible ici : https://library.duke.edu/ digitalcollections/wlmpc_wlmms01039/\#info [site consulté le 10/072019].

5. Robin Morgan, « No More Miss America!", New York Radical Women, 19-22 août 1968. Accessible ici : https://www.redstockings.org/index.php/no-more-miss-america [site consulté le 10/07/2019].

6. Ibidem. [souligné par mes soins]. 
7. Martha Boesing, «Rushing Headlong into the Fire at the Foot of the Mountain », Signs, $\mathrm{n}^{\circ} 4$, 1996, p. 1014.

8. Mary Daly, Gyn/Ecology : The Metaethics of Radical Feminism, Boston, Beacon Press, 1978.

9. Hélène Cixous, «Le rire de la méduse ", L'Arc, nº 61, 1975, pp. 39-64.

10. Courant du féminisme qui pense les différences d'attributs, de comportements, entre les femmes et les hommes.

11. Sue Ellen Case, Feminism and Theatre, Londres, R.-U., Palgrave Macmillan (réed.), 2008, p. 65.

12. Idem, Ibidem, p. 46.

13. Liz Kelly, Surviving Sexual Violence, Cambridge, R.-U., Polity Press, 1988, p. 76.

14. S. E. Case, op. cit. note 11, p. 67.

15. Laura Mulvey, «Visual pleasure and narrative cinema », Oxford Journals, $n^{\circ} 3$, automne 1975, pp. 6-18.

16. S. E. Case, op. cit. note 11, pp. xviii-xix.

17. Idem, Ibidem, pp. 68-69.

18. Charlotte Canning (dir.), Feminist Theatres in the USA: Staging Women's Experiences, New York, NY, Routledge, 1996, p. 47.

19. Idem, Ibidem, p. 68.

20. Sally Banes, Ibidem, p. 62.

21. Harriet Schiffer, Ibidem, p. 69.

22. C. Canning, Ibidem, p. 69.

23. S. E. Case, op. cit. note 11, pp. 68-69.

24. Roberta Sklar, dans Cornelia Brunner, «Roberta Sklar: Toward Creating A Women's Theatre ", The Drama Review, $n^{\circ}$ 2, 1980, pp. 33-34.

25. Helen Krich Chinoy, "Art Versus Business: The Challenge of Women in American Theatre », Women in American Theatre, 3rd edition, 1997, p. xxix.

26. Le Presidential Commission on the Status of Women (PCSW) est un comité consultatif réuni en 1961 par le président états-unien John F. Kennedy, sous la chaire d'Eleanor Roosevelt, pour enquêter sur les questions traitant de l'égalité des femmes et des hommes dans les milieux éducatifs et professionnels, ainsi que sur le plan légal.

27. S. E. Case, op. cit. note 11, p. 47.

28. Elaine Aston, "Foreward », dans Feminism and Theatre, p. xix.

29. Bertolt Brecht, Brecht on Theatre: The Development of an Aesthetic, 1957, Trad. John Willett, Londres, R.-U., Methuen, 1964, p. 192.

30. C. Canning, op. cit. note 18, p. 160.

31. Noelia Hernando-Real \& Barbara Ozieblo, «American Women Playwrights and Violence Against Women ", dans Noelia Hernando-Real \& Barbara Ozieblo, Performing Gender Violence: Plays by Contemporary American Women Dramatists, Londres, R.-U., Palgrave Macmillan, 2012, p. 37.

32. S. E. Case, op. cit. note 11, p. 116. On peut ici penser aux prix des billets d'entrée calculés au pro rata par la compagnie At the Foot of the Mountain, comme le témoigne sa directrice artistique Boesing. Voir « Rushing Headlong into the Fire at the Foot of the Mountain », p. 118.

33. Idem, Ibidem.

34. Ibid., p. 121. Voir Luce Irigaray, Ce sexe qui n'en est pas un, Paris, Éditions de Minuit, 1977.

35. Patti P. Gillespie, «A Feminist Theory of Theatre: Introduction », Women in American Theatre, p. 338.

36. Pour en lire plus à ce sujet, voir Feminist Theatrical Revisions of Classic Works : Critical Essays, Ed. Sharon Friedman, Jefferson, NC, McFarland, 2009.

37. Teresa de Lauretis, "Feminist Studies / Critical Studies: Issues, Terms, and Contexts", Feminist Studies / Critical Studies, Ed. Teresa de Lauretis, Bloomington, Indiana University Press, 1986, p. 11.

38. S. E. Case, op. cit. note 11, p. 132. 
39. Voir Judith Butler, Gender Trouble: Feminism and the Subversion of Identity, New York, NY, Routledge, 1990.

40. C. Canning, op. cit. note 18, p. 207.

41. Voir This Bridge Called My Back: Writings by Radical Women of Color, New York, Ed. Cherríe Moraga et Gloria Anzaldúa, Kitchen Table, Women of Color Press, 1983, et Bell Hooks, Feminist Theory from Margin to Center, 1984, Londres, R.-U., Pluto, 2000.

42. Judith Butler « Performative Acts and Gender Constitution: An Essay in Phenomenology and Feminist Theory ", Theatre Journal, $\mathrm{n}^{\circ}$ 4, 1988, pp. 519-531.

43. Kimberle Crenshaw, "Demarginalizing the Intersection of Race and Sex: A Black Feminist Critique of Antidiscrimination Doctrine, Feminist Theory and Antiracist Politics ", University of Chicago Legal Forum, $\mathrm{n}^{\circ}$ 1, 1989, pp. 139-166.

44. Susan Faludi, Backlash: The Undeclared War Against American Women, New York, Anchor Books, 1992.

45. Audre Lorde, «The Master's Tools Will Never Dismantle the Master's House ", op. cit. note 41, p. 99.

46. L'on peut penser ici à la publication de l'anthologie de monologues Out from Under: Texts by Women Performance Artists. Ed. Lenora Champagne, New York, NY, Theatre Communications Group, 1990 ou bien à l'étude fondamentale de seuls et seules en scène queer et intersectionelles de José Esteban Muñoz, Disidentifications : Queers of Color and the Performance of Politics Minneapolis, University of Minnesota Press, 1999.

47. Jill Dolan, «In Defense of the Discourse: Materialist Feminism, Postmodernism, Poststructuralism... And Theory ", The Drama Review, n 3, 1989, p. 50.

48. Judith Butler, «Imitation and Gender Insubordination », Literary Theory: An Anthology, Malden, Blackwell, 1998, p. 723.

49. Lynda Goldstein, «Raging in Tongues: Confession and Performance Art », Confessional Politics: Women's Sexual Self-Representations in Life Writing and Popular Media, Ed. Irene Gammel, Carbondale, Southern Illinois University Press, 1999, pp. 107-108.

50. Voir Teresa de Lauretis, «Eccentric Subjects: Feminist Theory and Historical Consciousness », Feminist Studies, $\mathrm{n}^{\circ}$ 1, 1990, pp. 115-150.

51. Holly Hughes, Clit Notes: A Sapphic Sampler, New York, Grove Press, 1996, p. 216.

52. J. Dolan, The Feminist Spectator as Critic, 2nd edition, Ann Arbor, University of Michigan Press, 2012, p. 70. Parmi d'autres lieux alternatifs « Off-Off Broadway» qui produisent notamment du théâtre féministe, queer et racisé, l'on peut citer : Women's Interart Center (1971-1980), fondé par Jacqueline Skiles; La MaMa Experimental Theatre Club (E.T.C.), fondé en 1961 par Ellen Stewart ; et Dixon Place, fondé en 1985 par Ellie Covan.

53. WOW Café Theatre, "Mission", https:// www.wowcafe.org/about/, [site consulté le 10/07/2019].

54. Pour en lire plus sur la subversion du langage par la performance, voir J. Butler, Excitable Speech: A Politics of the Performative, New York, Routledge, 1997.

55. L. Goldstein, op. cit. note 49, p. 108.

56. Monique Wittig, « La pensée straight », Questions Féministes, ${ }^{\circ}$ 7, 1980, p. 53.

57. Voir Dolan sur le cas spécifique de la «femme-butch » représentée dans des pièces féministes postmodernistes, The Feminist Spectator as Critic, pp. 76-77.

58. Agence fédérale chargée des allocations de subventions pour des actrices et acteurs de la culture.

59. George H. W. Bush, cité dans Richard Meyer, «Have You Heard the One about the Lesbian Who Goes to the Supreme Court? Holly Hughes and the Case against Censorship ", Theatre Journal, $\mathrm{n}^{\circ} 4,2000$, p. 543. Les trois autres artistes états-uniens du « NEA 4 » sont Karen Finley, John Fleck et Tim Miller. 
60. Pour en lire plus sur cette culture war, commencer par le discours notoire de Patrick Buchanan, candidat présidentiel paléoconservateur qui livre en 1992 «The Cultural War for the Soul of America »; afin de comprendre la tendance conservatrice du débat, lire en ligne https:// buchanan.org/blog/the-cultural-war-for-the-soul-of-america-149 [site consulté le 10/07/2019]. 61. Voir interview d'Holly Hughes dans « Speaking Freely : Interview with Holly Hughes » (2000), YouTube, téléchargé par Newseum, 26 octobre 2015, https://www.youtube.com/watch? $\mathrm{v}=$ BZAozLXPXS8, [site consulté le 10/07/2019].

62. J. Dolan, Utopia in Performance: Finding Hope at the Theatre, Ann Arbor, University of Michigan Press, 2005, p. 50. Pour visionner le seul-en-scène dans sa totalité, voir Hemispheric Institute of Performance and Politics, «Holly Hughes Preaching to the Perverted (1999)», https:// hemisphericinstitute.org/en/hidvl-collections/item/2709-holly-hughes-preaching-to-theperverted-1999.html [site consulté le 10/07/2019].

63. J. Dolan, Utopia in Performance, p. 49.

64. L. Goldstein, op. cit. note 49, pp. 112-113.

65. Christine M. Cooper, «Feminism and Eve Ensler's The Vagina Monologues », Signs, $\mathrm{n}^{\circ}$ 3, 2007, p. 741.

66. Camille Froidevaux-Metterie, Le corps des femmes: La bataille de l'intime, Paris, Philosophie magazine Éditeur, 2018, p. 13. Aux États-Unis, ce tournant est annoncé, par exemple, par la publication consécutive des ouvrages suivants à la frontière du champ de la théorie féministe et des genres littéraires du « self-help »/ « self-empowerment », fortement marqués par la tendance politique néolibérale. Je cite : Inga Muscio, Cunt: A Declaration of Independence, Seattle, WA, Seal Press, 1998 ; Carol Livoti \& Elizabeth Topp, Vaginas: An Owner's Manual, New York, Thunder's Mouth Press, 2004 ; Naomi Wolf, Vagina: A New Biography, Londres, R.-U., Virago Press, 2012 ; Emma L.E. Rees, The Vagina: A Literary and Cultural History, New York, Bloomsbury, 2013.

\section{RÉSUMÉS}

Un retour sur les débuts des théâtres féministes états-uniens permet une réhabilitation de l'histoire de ces théâtres, qui se sont construits en parallèle des mouvements pour les droits civiques à partir des années 1960. La pratique théâtrale se voit sollicitée comme vecteur de revendications politiques, et le plateau devient un espace de représentation des violences sociales et physiques/morales subies par des femmes-identifiées, au sein du milieu artistique, et bien au-delà. Des premières troupes féministes à l'émergence de pratiques solistes, le théâtre - de son écriture à sa mise en scène - traduit également des imaginaires alternatifs, utopiques, affranchis de violences et d'oppression. Développé à travers le prisme des théories critiques féministes, queer et intersectionnelles, ce travail expose les enjeux de ce moment artistique et convoque son héritage contemporain.

A look back at the origins of American feminist theatres, built in parallel with the civil rights movements as from the 1960s, allows the history of these theatres to be rehabilitated. Theatre practice developed into a vehicle for political demands, and the stage into a space for representing the social and physical/moral violence suffered by identified women in artistic circles and further afield. From the first feminist theatre companies to the emergence of individual practices, the theatre - from the writing of a play to its staging - also provides a channel for alternative, utopian imaginative worlds free from violence and oppression. 
Developed through the prism of feminist, queer and intersectional critical theories, this work reveals the issues that were at stake at that artistic moment and discusses its contemporary heritage.

\section{INDEX}

Keywords : theatre, performance, performing arts, alone on stage, United States, feminisms, gender, archives, violence, political arts, identified women, queer

Mots-clés : Théâtre, performance, spectacle vivant, seule en scène, Féminismes, Genre, Archives, Violences, Arts politiques, Femmes identifiées, Queer

Index géographique : États-Unis

\section{AUTEUR}

\section{MADELEINE PLANEIX-CROCKER}

Madeleine Planeix-Crocker (Los Angeles, 1993) est une curatrice-chercheuse franco-américaine. Spécialisée en politique culturelle (BA, Princeton University et Master spécialisé Médias, Art et Création, HEC Paris) et en études de genre et de performance (M2, EHESS), elle a commencé en 2019 à l'EHESS (CRAL) sa thèse consacrée à l'analyse des performances en commun programmées en institutions culturelles françaises à l'ère contemporaine, auprès d'Elizabeth Claire (EHESS, $\mathrm{CRH} / \mathrm{CNRS}$ ) et d'Esteban Buch (EHESS, CRAL). Elle est également curatrice d'un cycle de performance inclusif à Lafayette Anticipations - Fondation Galeries Lafayette, intitulé les "Warm Up Sessions". En 2017, elle a fondé un atelier théâtre à l'association Women Safe qui a fait l'objet d'étude de sa recherche-création de M2. En parallèle, Madeleine maintient sa pratique de metteuse en scène et de chorégraphe.

Madeleine Planeix-Crocker (Los Angeles, 1993) is a French-American curator-researcher. Specialising in cultural policy (BA, Princeton University and a Master's specialising in Media, Art and Creation, HEC Paris) and in gender and performance studies (M2, EHESS), she began her thesis in 2019 at EHESS (CRAL) supervised by Elizabeth Claire (EHESS, CRH/CNRS) and Esteban Buch (EHESS, CRAL). Her study analyses joint performances programmed in French cultural institutions in the contemporary era. She is also the curator of an inclusive performance cycle at Lafayette Anticipations - Fondation Galeries Lafayette, titled “Warm Up Sessions”. In 2017, she founded a theatre workshop at the Women Safe association, which was the subject of her M2 research and creation. Alongside these activities, Madeleine maintains her practice as a director and choreographer. 